\title{
ACQUISITION AND PROCESSING PROTOCOLS FOR UAV IMAGES: 3D MODELING OF HISTORICAL BUILDINGS USING PHOTOGRAMMETRY
}

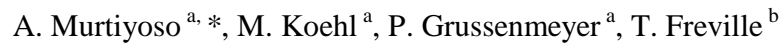 \\ ${ }^{a}$ Photogrammetry and Geomatics Group, ICube Laboratory UMR 7357, INSA Strasbourg, France - \\ (arnadi.murtiyoso, mathieu.koehl, pierre.grussenmeyer)@insa-strasbourg.fr \\ ${ }^{\mathrm{b}}$ Drone Alsace, Strasbourg, France - contact@ drone-alsace.fr
}

\section{Commission II}

KEY WORDS: UAV, documentation, photogrammetry, close range, protocols, heritage buildings

\begin{abstract}
:
Photogrammetry has seen an increase in the use of UAVs (Unmanned Aerial Vehicles) for both large and smaller scale cartography. The use of UAVs is also advantageous because it may be used for tasks requiring quick response, including in the case of the inspection and monitoring of buildings. The objective of the project is to study the acquisition and processing protocols which exist in the literature and to adapt them for UAV projects. This implies a study on the calibration of the sensors, flight planning, comparison of software solutions, data management, and analysis on the different products of a UAV project. Two historical buildings of the city of Strasbourg were used as case studies: a part of the Rohan Palace façade and the St-Pierre-le-Jeune Catholic church. In addition, a preliminary test was performed on the Josephine Pavilion. Two UAVs were used in this research; namely the Sensefly Albris and the DJI Phantom 3 Professional. The experiments have shown that the calibration parameters tend to be unstable for small sensors. Furthermore, the dense matching of images remains a particular problem to address in a close range photogrammetry project, more so in the presence of noise on the images. Data management in cases where the number of images is high is also very important. The UAV is nevertheless a suitable solution for the surveying and recording of historical buildings because it is able to take images from points of view which are normally inaccessible to classical terrestrial techniques.
\end{abstract}

\section{INTRODUCTION}

Aerial photography has a long history of applications in cartography. It has a very large range of applications and can be used to minimize work on the ground. Photogrammetry in turn is a standard tool often used for large scale mapping applications. This technique enables the user to obtain 3D data of the zone of interest, and eventually may also be used to produce orthophotos. Photogrammetry has also seen an increase in the use of UAVs (Unmanned Aerial Vehicles) for smaller scale cartography. Their use is also advantageous because it may be used for tasks requiring quick response, including in the case of the inspection and monitoring of buildings.

Originally a military conception, the UAV has seen a significant shift towards civilian use. The spread of UAVs complements the existing terrestrial image based techniques in enabling a close range aerial photogrammetry operation. Coupled with developments in sensors and computing power, this type of surveying becomes a powerful solution for various uses. Heritage documentation naturally benefits from these developments, as it complements terrestrial techniques (Barsanti et al., 2014; Nex and Remondino, 2014). Some examples in this domain can be seen in Achille et al. (2015), Alidoost and Arefi (2015), Baiocchi et al. (2013), Cefalu et al. (2013), Fritsch et al. (2013a), and Reich et al. (2012).

In the remote sensing domain, the on-board sensor's optics plays an important role. Several UAV manufacturers have tried to integrate better quality lenses, but the platform's payload remains an important issue. On the other hand, the field of computer vision has largely facilitated and complemented classical photogrammetry. As explained in Chiabrando et al. (2015) and Remondino et al. (2012), the typical workflow involves automatic tie point feature extraction and matching. This is often followed by robust outlier detection and elimination and bundle block adjustment in order to retrieve the position and orientation of each camera station.

In this research, several studies were performed, notably on the calibration of the sensors, flight planning, comparison of software solutions, data management, and analysis on the different products of a UAV project. Furthermore, a geometric and visual analysis of the results was performed.

Two historical buildings of the city of Strasbourg were used as case studies: a part of the Rohan Palace façade and the StPierre-le-Jeune Catholic church. In addition, a preliminary test was performed on the Josephine Pavilion. The data acquisition was performed mainly using the UAV photogrammetry technique. A terrestrial laser scanner (TLS) was also employed to acquire reference data. These buildings were chosen with the recommendation of the Strasbourg City Council by taking into account the different challenges related to both the acquisition and the processing. It is also a pilot project for the recording of heritage with the final objective of integrating them within the 3D layer of the city's GIS (Geographic Information System).

\section{RELATED WORKS}

Several categorizations of UAVs were given by Colomina and Molina (2014), Fritsch et al. (2013b), Nex and Remondino (2014), and Remondino et al. (2011). However, a more practical classification of UAV types based on their physical

\footnotetext{
* Corresponding author
} 
structure can also be derived (Achille et al., 2015; Nex and Remondino, 2014; Remondino et al., 2012):

1. Lighter than air platforms: such as balloons and kites. This category is low-cost but is more difficult to control due to its low wind resistance and low velocity.

2. Fixed-wing platforms: with the capability of covering a large surface but may be limited in payload as well as wind resistance. The fixed-wing UAV is suited for larger-scale mapping resembling classical smallformat aerial photogrammetry.

3. Rotary-wing platforms: either with a single or multiple rotors. This type of UAV has a larger payload and wind resistance, but its surface coverage can be significantly lower than that of the fixed-wing type.

Furthermore, several acquisition and processing procedures already exist in the literature. The CIPA Heritage Documentation's 3x3 rules have existed since 1994 with several updates (Grussenmeyer et al., 2002). The latest version can be consulted in the CIPA website (http://cipa.icomos.org/). Another protocol called TAPENADE (Tools and Acquisition Protocols for Enhancing Artefacts Documentation) was also developed especially for the documentation of cultural heritage in mind (Nony et al., 2012; Pierrot-Deseilligny et al., 2011). The University of Stuttgart's Institute for Photogrammetry also developed their protocol (Wenzel et al., 2013), "One panorama each step", which was already tested on UAV datasets.

In terms of existing dense matching algorithms, most 3D reconstruction software which is based on images has their own algorithm for the generation of a dense point cloud. Remondino et al. (2013) and more recently Remondino et al. (2014) have tried to classify the different existing approaches to image matching. The most basic classification is between the matching of features (i.e. comparison of descriptors) and the matching of grayscale value within a pre-determined search window. Once the correspondence is done, a simple mathematical calculation is performed in order to determine the coordinates of the object on the object space. The matching of features is otherwise called Feature-Based Matching (FBM) while the other classification is called Area-Based Matching (ABM) (Remondino et al., 2013). FBM is often employed to help the orientation process, while ABM is used in dense matching.

Several software solutions were also tested in this research, both in terms of image orientation and dense matching. This includes Agisoft Photoscan (PS), Pix4D (P4D), Photomodeler by EosSystems (PM), and Micmac by IGN (MM).

P4D and PS are two commercial solutions with a rather blackbox nature. However, concurrent with the results of Remondino et al. (2014), PS most probably performs a modified SGM (Semi-Global Matching) (Hirschmüller, 2011) of stereo pairs to generate depth maps, and then employs epipolar constraints at the end of this process to filter the results. P4D may have used a similar albeit different approach. Meanwhile PM is well-known by the architecture and archaeology community for performing 3D image-based measurements (Grussenmeyer et al., 2002). PM has since added a dense matching module based on stereo-pairs and more recently on a multi-view geometry. Finally, MM uses a multi-resolution and multi-image approach to dense matching (Pierrot-Deseilligny and Paparoditis, 2006).

\section{TOOLS AND METHODS}

\subsection{Tools Employed}

The first UAV used in this project was a multi-copter rotary wing platform manufactured by the company Sensefly and called Albris since April 2016 (previously known as Exom). The product first appeared in the market in May 2015 and was aimed to perform close-range high resolution inspections, mapping, and 3D modelling. It is equipped with multiple sensors, including a still 38 megapixels (MP) camera, a thermal and a video camera. Several ultrasonic sensors give an approximate distance measurement of its surroundings, enabling it to fly on a set distance from an object. The still camera itself is furnished with an $8 \mathrm{~mm}$ lens and a $10 \times 7.5 \mathrm{~mm}$ sensor. This specification theoretically enables a GSD (Ground Sampling Distance) of up to $1 \mathrm{~mm}$ at a distance of 6 meters. This UAV has a payload of $1.8 \mathrm{~kg}$. This UAV was chosen in order to test its state-of-art sensorial capabilities for use in close range photogrammetry.

Another UAV used was the DJI Phantom 3 Professional, also a multi-copter rotary wing type. This UAV was first released also in May 2015. The Phantom 3 is lightweight and relatively lowcost, but it is not geared for dedicated close-range inspection tasks. Unlike the previous versions, the Phantom 3 is no longer equipped with a fish-eye lens camera. This could potentially yield a better geometric result, but the sensor itself remains very small compared to most terrestrial cameras. It is equipped with a $4 \mathrm{~mm}$ lens and a 6.5 x $5 \mathrm{~mm}$ sensor, with a $1.3 \mathrm{~kg}$ payload.

A TLS was also used to acquire the point cloud of both case studies. In this case the phase-based TLS FARO Focus $3 D$ $X 330$ was used. The scanner is associated with the software FaroScene which performs the point cloud registration and georeferencing. The registration process may be aided by the use of spheres which are automatically detected in FaroScene. A manual registration of the point clouds is also possible.

\subsection{Acquisition Protocol}

In order not only to strengthen the image network geometry but also to better cover hidden parts, a flight taking perpendicular images was immediately followed by four oblique flights with the cameras tilted to the right, left, up, and down (see Murtiyoso et al., (2016) for more details). The configuration of flight was not changed for all five flights in order to emulate a highly overlapping and convergent geometry. The primary downfall of this method is that the same flight must be performed five times for the five sensor attitudes. This increases flight time and therefore also poses problems in regards to battery issues and image texture homogeneity, and eventually also the calibration stability of the sensors.

\subsection{Processing Strategy}

In principle, the photogrammetric workflow starts with the feature matching and is followed by external orientation (with absolute orientation included) and dense matching based on the pixels. Indeed, in many software packages this workflow is already well defined. The user can generate the products simply by following the given workflow. In open source solutions this is sometimes less clear, but often times the user is directed towards forums in which questions, responses, and information regarding the software can be found. In both cases, the general photogrammetric workflow remains similar. 


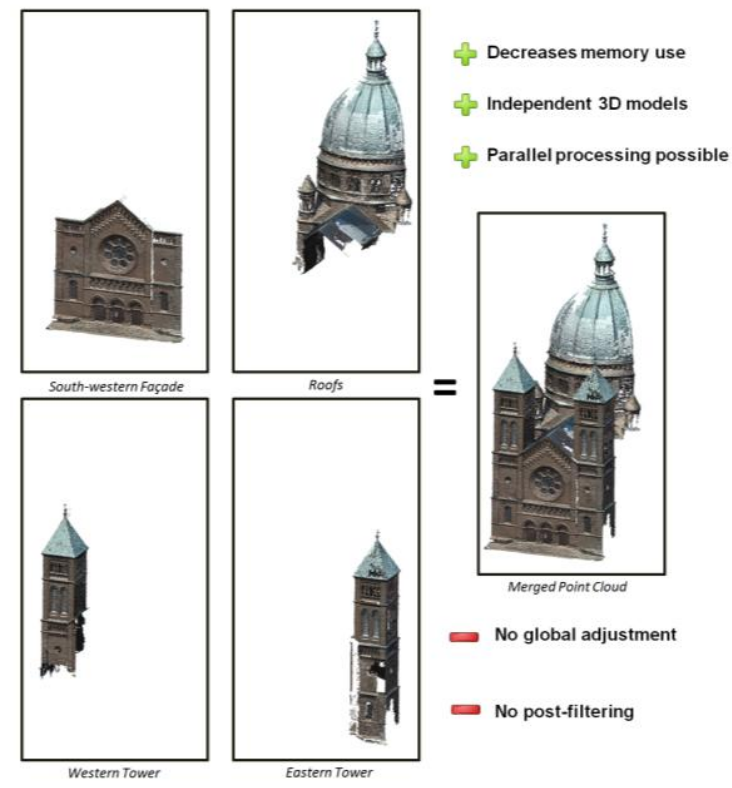

Figure 1. Illustration of the project decomposition principle used in this study, using the example of the south-western façade of the St-Pierre-le-Jeune dataset.

However, in the case of a project with large number of images $(1,000+)$, a form of project management is in order. A simultaneous processing of all images will require large amounts of resources (processor, memory, etc.). The strategy proposed in this study was used in the processing of the StPierre-le-Jeune dataset, which has more than 2,500 images. Note that the computer used in the data processing possesses a 32 GB RAM, 16 core Intel(R)Xeon(R) processor $(2 \mathrm{GHz})$, and an NVIDIA Quadro 40004 GB graphics card.

In this method, the project is decomposed into several smaller clusters. The proposed processing strategy is quite straightforward and follows the illustration given in Figure 1. This decomposition has the advantage of facilitating the management of processing resources and enables the project to advance in parallel with the data acquisition in the field. Therefore the clusters can be processed individually without having to wait until data acquisition is finished. However, this strategy does not permit the use of a global adjustment for the whole project. Moreover the generated point cloud is not filtered, which may result in redundant points. The radiometric adjustment is performed after the point clouds are assembled and meshed, as to render a more homogeneous texture.

\section{RESULTS AND ANALYSIS}

\subsection{Sensor Calibration}

The calibration was performed for the two sensors integrated within the two UAVs used in this project. Several software programmes were used to perform the calibration in order to compare the results: PM, PS, and the Apero module of the MM. For the sensor in Albris, the calibration was also performed using PostFlightTerra3D which is a limited version of the software P4D. Due to limitations in licensing options, this is only possible to do with the Albris. However, it is also possible to compare the results with the values given by $\mathrm{P} 4 \mathrm{D}$.

During the calibration, the only criterion which was not respected was the rotation of the sensor into the portrait mode (as recommended in Luhmann et al. (2015), Remondino (2014) and Remondino and Fraser (2006)). The two UAVs are equipped with gimbals which prevent the sensor from deviating from a horizontal position. As a result, the portrait images were not taken. This may eventually generate a correlation between the parameters $\mathrm{P}_{1}$ and $\mathrm{P}_{2}$, which further means that these two values should be deactivated during the calibration calculations.

As regards to the radial distortion, Phantom 3 shows a strong correlation between the $\mathrm{K}_{2}$ and $\mathrm{K}_{3}$ values of up to $96.9 \%$. Furthermore, the standard deviation of the $K_{2}$ value is very high (1.6 times that of the value itself). Fraser (2013) mentioned that for low precision sensors, $K_{3}$ and eventually $K_{2}$ may be deactivated. On the other hand, Albris gave stable results between the different solutions. See Murtiyoso et al. (2017) for more discussion regarding the radial distortion curves. Some possible sources of error during the process of calibration:

1. The quality of the sensor: the UAVs used in this project are equipped with small sensors. Phantom 3 has a $6.5 \times 5 \mathrm{~mm}$ sensor while Albris is equipped with a slightly larger $10 \times 7.5 \mathrm{~mm}$ sensor. These types of sensors are not designed for high precision use and were rather conceived for visual works. Indeed, the radial distortion value can theoretically reach 40 pixels for Phantom 3 and 90 pixels for Albris (for points located on the image edges).

2. Sensor instability: Being small sensors, their internal geometry is easily changed due to movements or changes in imaging conditions. The photographs were also taken using the auto-focus mode; this gave more instability on the obtained values because each image taken would have a slightly different focal length.

3. Quality of the images: In this study, the calibration was done using coded targets which were detected automatically by PS and PM. However, the other solutions didn't have this capability of coded target automatic detection. The quality of the image is therefore very important when identifying the points manually. Similarly, the feature matching which is used to strengthen the quality of the calibration is also influenced by this fact. In the case of the Phantom 3, this factor is limited by the image size of 12 MP. For Albris, noises were present on the images on all datasets. This noise may therefore influence the quality of the calibration.

\subsection{Preliminary Test: the Josephine Pavilion}

A preliminary test was performed on the Josephine Pavilion, a $19^{\text {th }}$ century building located inside the Orangerie Park in the city of Strasbourg. The building was photographed using the UAV DJI Phantom 3. The images were taken from an average distance of 8 meters in such way as to cover the four facades. Nadiral images were also taken around the object. Being a test project, only several control points were measured on the face by using a total station with the help of the city of Strasbourg. The particularity of this building is its material; the north (back) façade is made from red sandstone, typical of the Alsace region while the south (front) façade is a relatively smooth wall painted in beige, giving little texture.

Several preliminary analyses were taken from this pilot project. The first concerns the importance of image overlap of the object; indeed echoing the need for detailed images with large percentage of overlap mentioned in the existing protocols. A 
remark was made to acquire controlled oblique images in further projects in order not only to strengthen the image network geometry but also to better cover hidden parts (window sills, awnings, balcony, etc.).

Furthermore, the problem of the object in question's material as regards to its influence on the dense matching is also important to note. In the case of the pavilion, a textureless material hinders a proper pixel-by-pixel search for correspondence. Particular strategies should be taken to address this problem. One strategy that can be employed involves changing the resolution of the images used in the dense matching; bearing in mind that in this case a better resolution matching does not necessarily produce a better point cloud. Another solution involves changing the correlation coefficient threshold to allow more points (which may however be noisy) to be detected.

\subsection{First Case Study: the Rohan Palace}

The Rohan Palace is a historical landmark of the city of Strasbourg dating to the $18^{\text {th }}$ century. Located next to the cathedral, the palace was built for the Cardinal Rohan between 1732 and 1742 and housed several French sovereigns during their visits to Strasbourg. Today it is used by three museums, all managed by the city's administration. In regards to the material, the palace is made of yellow sandstone. For this project, only the central façade overlooking the River Ill was photographed. The dimension of this façade is approximately $14 \times 20$ meters.

In this project, the Sensefly Albris was used to acquire the images. Using its capability to fix object-to-camera distances, the UAV was flown at a fixed approximate distance of 5 meters from the façade. Overall, 555 images were taken for this dataset using the Albris. In addition, 13 control and check points scattered on the façade were measured using a total station using the spatial intersection method in order to be able to assess the precision and accuracy of the results. The 6 control points are placed on the limits of the flight zone following the classical aerial photogrammetry configuration. The remaining 7 points were used as check points, and are scattered evenly on the façade to represent changes of reliefs.

Despite the theoretical GSD of $0.9 \mathrm{~mm}$, here the precision of the aerotriangulation for all four solutions was on average $9 \mathrm{~mm}$ while the check point accuracy was $7 \mathrm{~mm}$. It should be noted that the control and check points used were detail points (window edges, bricks, etc.) and not well defined targets. The precision and accuracy depend therefore on the quality of point measurement. This however, was hampered by the noisy quality of the Albris' images. This noise problem has been acknowledged by Sensefly and an improvement of sensor quality is expected sometime soon.

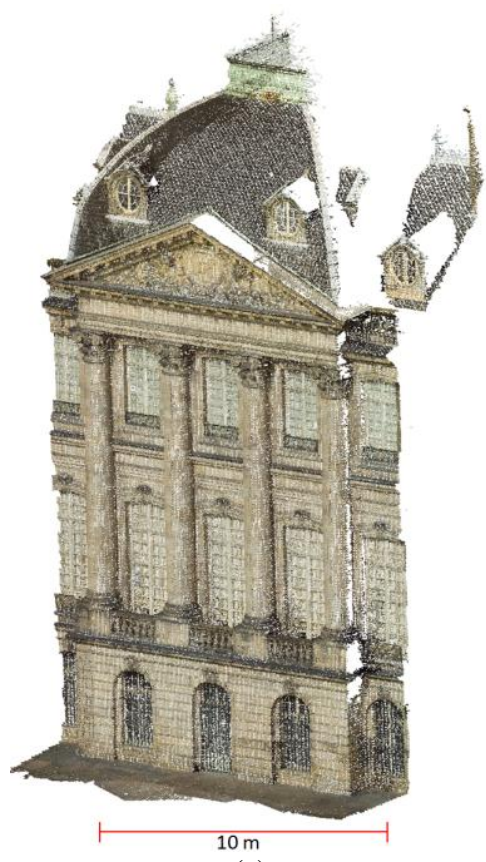

(a)

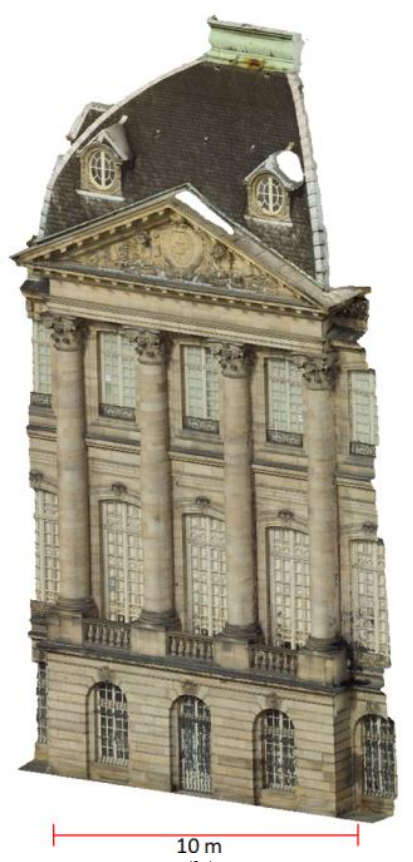

(b)

Figure 2. Results of the dense matching (a) and the meshed and textured model (b) of the Rohan dataset.

\begin{tabular}{|c|c|c|c|c|}
\hline & $\boldsymbol{P S}$ & $\boldsymbol{P 4 D}$ & $\boldsymbol{M M}$ & $\boldsymbol{P M}$ \\
\hline Software version & 1.1 .6 & 2.1 .53 & Rev5999(201015) & 2016.2 .1 .2024 \\
\hline Preset name & Medium & Quarter Resolution & C3DC MicMac & N/A \\
\hline Input images resampling & 0.25 & 0.25 & 0.25 & 0.25 \\
\hline Point cloud downsampling & Unknown & 4 (“Optimal”) & 4 & 4 (Level 2) \\
\hline Post-matching filtering & "Aggressive” & $\begin{array}{c}\text { Coplanarity-based, } \\
\text { 3 rays/point }\end{array}$ & $\begin{array}{c}\text { Pre-matching, based } \\
\text { on best master and } \\
\text { secondary images } \\
\text { (AperoChImSecMM) }\end{array}$ & $\begin{array}{c}\text { Coplanarity-based, } \\
3 \text { rays/point }\end{array}$ \\
\hline Mean error $(\mathbf{m m})$ & 0 & 2 & 2 & 4 \\
\hline $\boldsymbol{\sigma}(\mathbf{m m})$ & 11 & 10 & 11 & 13 \\
\hline
\end{tabular}

Table 1. Dense matching settings for the Rohan Albris dataset and the results of its comparion to laser scanner data. 
As regards to the dense point cloud, the result of the dense matching process is shown in Figure 2 while the matching parameters employed for the four dense matching algorithms tested are shown by Table 1 . The matching settings used in each algorithm correspond to the resampling of the input images to a quarter of their original resolutions. Note that this resolution setting is often employed in dense matching solutions in order to give the users a compromise between quality and processing time. The application of oblique images had effectively covered several difficult places such as the balcony and the upper part of the frieze or the lower part of the pediment above it. However, the question of image noise still posed a problem. Indeed, a test performed with matching using half resolution images generated a sparser point cloud with many holes. On the contrary, a more complete point cloud was acquired using a lower resolution setting. This problem is most probably caused once again by the correlation coefficient threshold; in the higher resolution setting the algorithm calculates denser points which it assumes are noises and therefore deletes, leaving holes in the resulting point cloud.

Furthermore, in order to validate the accuracy of the dense matching results, a terrestrial laser scanning survey was performed on the façade concerned. This was done using a Faro Focus $3 D$ and the resulting point cloud has a resolution of about 6 to $8 \mathrm{~mm}$. Evaluation was performed for a common portion of each result of the photogrammetric dense matching using the laser scanner point cloud as reference. The statistical values concerning these comparisons can also be seen in Table 1. All solutions gave standard deviations of around $10 \mathrm{~mm}$ compared to the reference. This corresponds more or less to the theoretical resolution of the point cloud at this image pyramidal level of matching (around $14 \mathrm{~mm}$ ).

Larger histogram dispersion can be observed in PM's results, which indicates a noisier point cloud. More holes are also observed in this point cloud. PS and P4D gave more homogeneous i.e. less noisy results, with PS generating slightly more error. In addition, MM created more holes in general compared to P4D and PS, which may be related to the default correlation coefficient threshold in its semi-automatic C3DC matching mode.

\subsection{Second Case Study: St-Pierre-le-Jeune Catholic Church}

The St-Pierre-le-Jeune Catholic Church was built in Strasbourg during the German era between 1889 and 1893. It is an example of neo-Romanesque architecture crowned by a $50 \mathrm{~m}$ high and $19 \mathrm{~m}$ wide dome. Both the Sensefly Albris and the DJI Phantom 3 were used to photograph this building. The Albris was used to take high resolution images of the principal façade, while the Phantom 3 was used to complete the rest of the building. In addition, due to the presence of some vegetation around the object, several terrestrial photographs were also taken using a standard DSLR camera with an $18 \mathrm{~mm}$ lens. In total 2,755 images were taken for this dataset.

The images from Albris were taken from a distance of $8 \mathrm{~m}$ from the façade, which brings its theoretical GSD to $1.4 \mathrm{~mm}$. The object-to-camera distance for the Phantom 3 was less fixed and ranged between 10 to $15 \mathrm{~m}$. This gives a value of theoretical GSD for the Phantom 3 of around $5 \mathrm{~mm}$ in average. Control points were also measured around the church. A traverse network was established around the building which is attached to the French national coordinate system. The photogrammetric control points were measured from these traverse points on the façades of the church.

The aerotriangulation gave several interesting points. First of all, the main façade which was photographed using the Albris gave an average error of $7 \mathrm{~mm}$ for the four software solutions tested. It should be noted that each control point measurement was performed independently in each software solution. This means that accidental error can be an important factor for the value of precision given by each algorithm. Apart from PM, the other three solutions also performed re-projections of control points on the images based on a prior approximate orientation using the minimum requirement of three control points. The role of the automatic tie point matching algorithm used by each solution is therefore also important.

It is also interesting to compare this value with the aerotriangulation result from the Rohan dataset. The two objects are similar in the sense that both are façades. Here the aerotriangulation result of the St-Pierre dataset is of the same order to the one obtained from the Rohan one. However, the object-to-camera distance in this case is almost two times that of Rohan. The theoretical GSD of the St-Pierre dataset is therefore higher ( $1.4 \mathrm{~mm}$ compared to Rohan's $0.9 \mathrm{~mm}$ ).

As the focal length of the sensor remains the same between the two projects, a better aerotriangulation result is expected from the Rohan Palace dataset. However, this is not the case as both results are similar (in the order of millimetres). A main cause to this anomaly can be the quality of the images themselves. As previously cited, the UAV Albris used in this project had problems regarding image noise. Indeed, the images on the Rohan dataset were noisier than those from the St-Pierre dataset, which may be explained by the difference in average object-to-camera distance. This phenomenon brings into question the interest of taking close range images using this type of UAV, even if they are marketed as close range inspection drones. Given that the Albris is supposed to be able to generate $38 \mathrm{MP}$ images, this problem regarding image noise is particularly important to be taken into account.

The Albris' aerotriangulation error is also much higher (up to 9 times higher) than the expected value of around $0.8 \mathrm{~mm}$, based on the average GSD and taking into account manual measurement error. The Phantom 3 fared better, with a value of aerotriangulation error of $14 \mathrm{~mm}$ compared to its expected value of around $5 \mathrm{~mm}$. In any case, using the current flight settings, a centimetric precision is attainable using both UAVs. However, it is worth noting that although theoretically in photogrammetry the precision can be increased by changing the GSD, in this case it is shown that it is nevertheless limited by the quality of the sensor. In terms of precision for the fusion of the different clusters, P4D gave a 3D conformal transformation error of $19 \mathrm{~mm}$ while PS gave a value of $17 \mathrm{~mm}$.

Results for the dense matching and meshing process can be seen in Figure 3. The dense matching process for the principal façade (imaged using Albris) was performed using all four software solutions (Table 2), while the rest of the church which was photographed by the Phantom 3 was processed using only PS and P4D (Table 3). Due to their difference in image resolution, Albris generated a much denser point cloud than Phantom 3. In general, when comparing the dense matching algorithms visually, a similar analysis to that of the Rohan dataset can be obtained. PS and P4D generated more homogeneous and complete point clouds compared to MM and PM. 


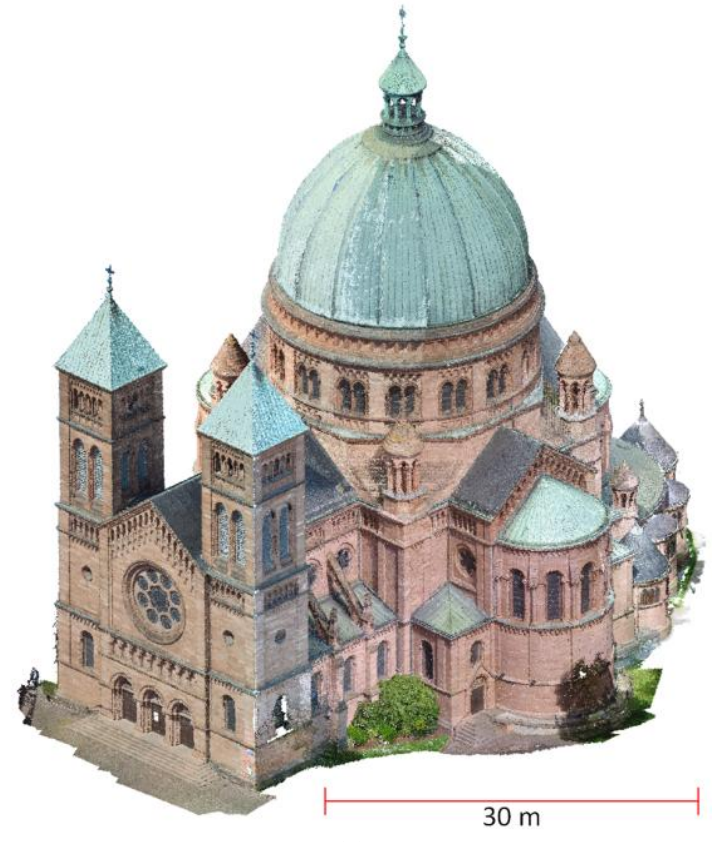

(a)

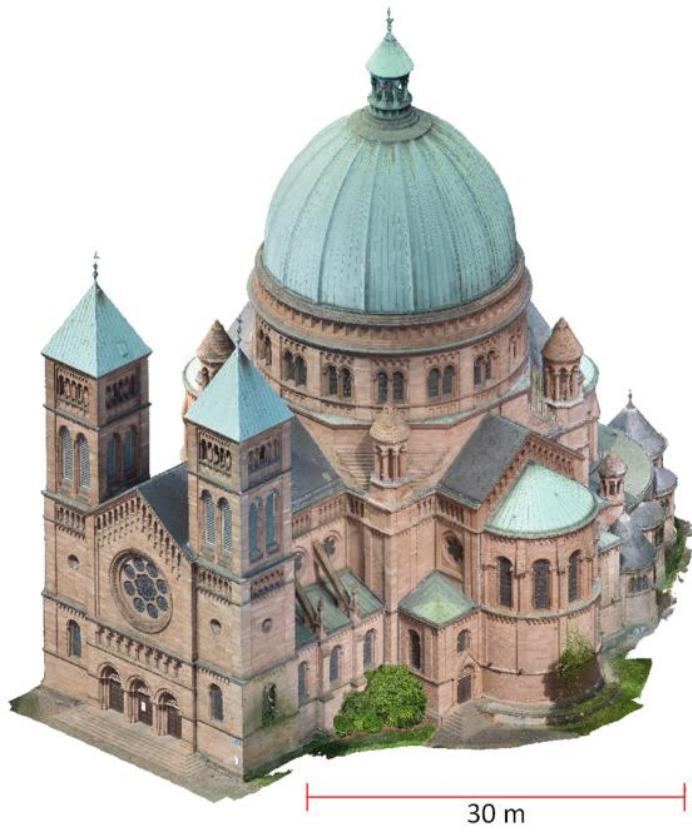

(b)

Figure 3. Final merged point cloud (a) and its meshed and textured model (b) of the St-Pierre dataset (2,700 combined images of Phantom 3, Albris, and terrestrial photos). Note the result of the radiometric compensation in (b).

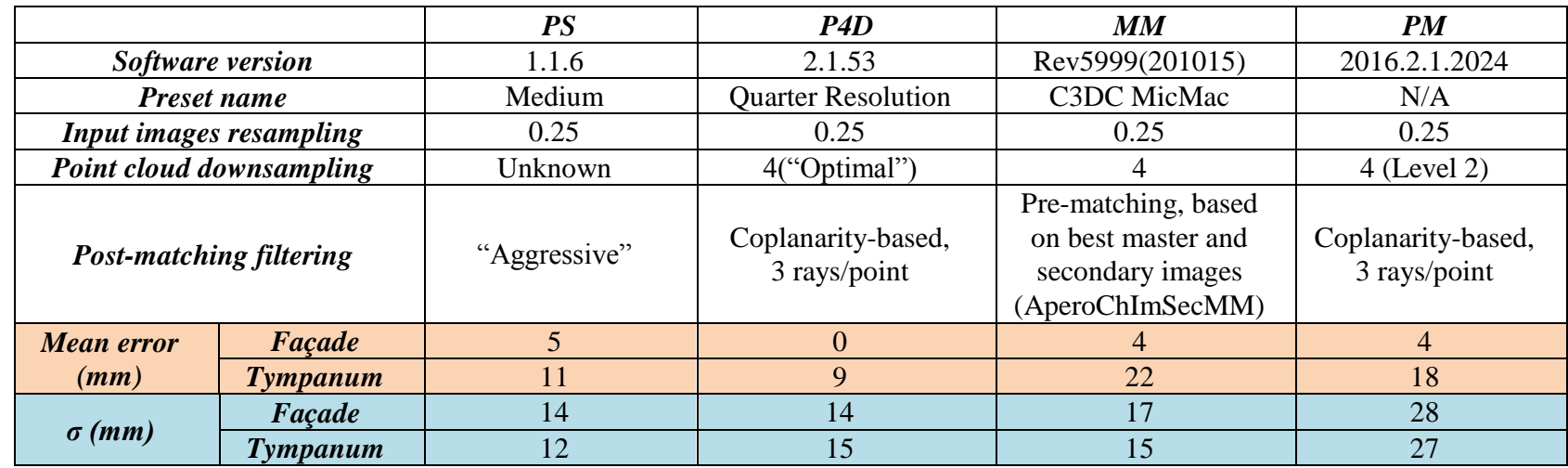

Table 2. Dense matching settings for the St-Pierre Albris dataset and the results of its comparion to laser scanner data.

\begin{tabular}{|c|c|c|c|}
\hline & & $P S$ & $P 4 D$ \\
\hline \multicolumn{2}{|c|}{ Software version } & 1.1 .6 & 2.1 .53 \\
\hline \multicolumn{2}{|c|}{ Preset name } & Medium & Quarter Resolution \\
\hline \multicolumn{2}{|c|}{ Input images resampling } & 0.25 & 0.25 \\
\hline \multicolumn{2}{|c|}{ Point cloud downsampling } & Unknown & 4("'Optimal”) \\
\hline \multicolumn{2}{|c|}{ Post-matching filtering } & "Aggressive" & $\begin{array}{c}\text { Coplanarity-based, } \\
3 \text { rays/point }\end{array}$ \\
\hline \multirow{2}{*}{$\begin{array}{l}\text { Mean error } \\
\quad(\mathrm{mm})\end{array}$} & Door & 14 & 19 \\
\hline & Gargoyle & 7 & 0 \\
\hline \multirow{2}{*}{$\sigma(m m)$} & Door & 14 & 14 \\
\hline & Gargoyle & 26 & 20 \\
\hline
\end{tabular}

Table 3. Dense matching settings for the St-Pierre Phantom 3 dataset and the results of its comparion to laser scanner data.

A laser scanning survey was also conducted on the main and south-east façades, in order to compare the results of the dense matching process for both UAVs. The same laser scanner, Faro Focus $3 D$, was used for this purpose. Four samples, two each from the results of each drone, were analysed (Figure 4). The statistical values for these comparisons are also shown in Tables 2 and 3 respectively. For the main façade, photographed by Albris, all solutions except PM gave on average a standard deviation value of around $15 \mathrm{~mm}$. PM is shown to have a higher standard deviation of up to $28 \mathrm{~mm}$. This shows a large dispersion in the resulting point cloud generated by $\mathrm{PM}$, which can be interpreted as an important presence of point cloud noise. MM registered a moderate standard deviation, which is without doubt caused by the systematic error observed on the lower part of the façade (the tympanums). A determining factor in this error is the quality of image orientation. Note that the expected theoretical resolution of point clouds generated by these settings is $22 \mathrm{~mm}$, so technically all results still fall within the set tolerance. In addition, all four solutions gave very small mean error values between 0 and $5 \mathrm{~mm}$. 


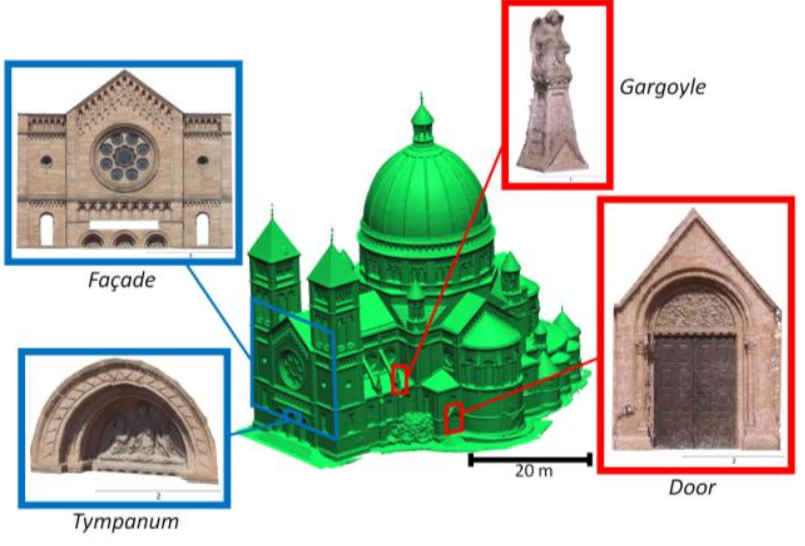

Figure 4. The four samples within the St-Pierre dataset which were compared against the TLS data. The blue squares indicate acquisition by Albris, while red squares indicate acquisition by Phantom 3.

In order to evaluate the result in a smaller scale, the point cloud of the main façade was segmented to extract the central tympanum. A slight systematic error amounting to $17 \mathrm{~mm}$ is observed in MM, while a similar standard deviation value as the façade is observed on PM. PS and P4D gave similar results, consistent with the point cloud for the Rohan dataset. The mean error in the tympanum is higher than the values for the entire façade for all solutions, which may be explained by the lack of points on the higher parts of the tympanum in the laser scanner point cloud. PM showed a larger dispersion up to $27 \mathrm{~mm}$ while the other solutions fall on the average value of $13 \mathrm{~mm}$.

In regards to the comparison between the point clouds generated from Phantom 3 images and the laser scanner data, a systematic error is observed on the two results, as shown by the mean error values. However, this may be caused by the quality of the reference point cloud, which was obtained from only one station. Furthermore, the door is also situated at the edges of the laser scanner point cloud, which further explains the existence of systematic error. Both standard deviations are of a similar order of $14 \mathrm{~mm}$, which is well within the expected theoretical resolution for this setting of dense matching.

One of the two gargoyles above a flying buttress on the southeastern façade was also analyzed. This object is situated right in front of a laser scanning station, which influences the minimal mean error of both software solutions employed. In contrast, the values of the standard deviation are high, up to 26 $\mathrm{mm}$. This may be due to the lack of points on the back of the gargoyle in the laser scanner data. The form of the object is also more complex, which implies that in order to obtain better results a dedicated processing should be performed for this level of detail. However, the value of the standard deviation is still within the range of the theoretical resolution of the point cloud.

\section{CONCLUSIONS}

Several experiments performed during the calibration stage showed that the interior parameters given by the UAV manufacturers are not always stable. Indeed, the calibration takes into account the variations in geometry as well as the stability of the sensor. Several experiments have also shown that the calibration parameters given by the manufacturers are not necessarily correct. Pre-project calibration is therefore always advised. If anything, it serves as good approximate values for the self-calibration process.

The dense matching of images remains a particular problem to address in a close range photogrammetry project, more so in the presence of noises on the images. The texture of the object plays a very important role, notably for the painted façades such as the case with the Josephine Pavilion. Therefore, a particular strategy should be envisaged to address these questions depending on the case. For the St-Pierre-le-Jeune dataset the quality of dense matching is strongly related to the computing power and internal memory of the machine. Data management in these cases where the number of images is high becomes very important.

Albris is an interesting UAV for close range inspections. With all of the on-board sensors, this UAV has the potential to become a very powerful inspection tool. This is also thanks to its capability to measure its camera-to-object distance. This function is very useful in close range photogrammetry as it enables the image to keep its GSD virtually constant. However, not withstanding all of its advantages, this UAV was not ready for use as of the time this research was conducted. The quality of the sensor needs to be improved to reduce the image noise. The noise in turn reduces the quality of the dense matching. Also in regards to its navigation system, many bugs and problems were still apparent during our experiments. DJI Phantom 3 on the other hand, although not especially designed for 3D modelling and close range inspection (as seen by the results of the calibration), is nevertheless a very stable UAV. It has also succeeded to generate a point cloud with centimetric precision without much problem.

In regards to the software solutions tested, P4D and PS generated relatively precise point clouds. However as discussed in Murtiyoso et al. (2016), there is a possibility that these algorithms perform a post-processing in order to give a more homogeneous point cloud. As their algorithms are of a blackbox nature, it is difficult to ascertain, nevertheless caution is advised for high-precision projects. Among the four algorithms tested, PM is marked with its focus on classical photogrammetric approaches. However, the dense matching module of PM still has several shortcomings compared to other software. Finally, MM gave precise results both in terms of aerotriangulation and dense matching. Its parameters are also easily modifiable. It is therefore a very useful open-source alternative to the existing commercial solutions.

During this project, several analyses were performed on historical buildings in the city of Strasbourg. CIPA explained that such type of documentation is important, because a monument can only be restored and conserved when it is well documented and measured in a repetitive manner and that these information are processed in an organized and structured system.

\section{ACKNOWLEDGEMENTS}

The research presented in this paper is the result of collaboration between INSA Strasbourg's Photogrammetry and Geomatics Group and the company Drone Alsace (www.dronealsace.fr/). The final 3D models (meshed and textured) can be viewed through the following Sketchfab links: https://skfb.ly/KE9B (Josephine Pavilion), https://skfb.ly/MsAo (Rohan Palace) and https://skfb.ly/PtQF (St-Pierre-le-Jeune church). These links were last accessed on 22 March 2017. 


\section{REFERENCES}

Achille, C., Adami, A., Chiarini, S., Cremonesi, S., Fassi, F., Fregonese, L., Taffurelli, L., 2015. UAV-based photogrammetry and integrated technologies for architectural applications - methodological strategies for the after-quake survey of vertical structures in Mantua (Italy). Sensors (Switzerland) 15, 15520-15539.

Alidoost, F., Arefi, H., 2015. An image-based technique for 3D building reconstruction using multi-view UAV images. ISPRS International Archives of the Photogrammetry, Remote Sensing and Spatial Information Sciences XL-1/W5, 43-46.

Baiocchi, V., Dominici, D., Mormile, M., 2013. UAV application in post-seismic environment. ISPRS - International Archives of the Photogrammetry, Remote Sensing and Spatial Information Sciences XL-1/W2, 21-25.

Barsanti, S.G., Remondino, F., Fenández-Palacios, B.J., Visintini, D., 2014. Critical factors and guidelines for 3D surveying and modelling in Cultural Heritage. International Journal of Heritage in the Digital Era 3, 141-158.

Cefalu, A., Abdel-wahab, M., Peter, M., Wenzel, K., Fritsch, D., 2013. Image based 3D Reconstruction in Cultural Heritage Preservation. Proceedings of the 10th International Conference on Informatics in Control, Automation and Robotics 201-205.

Chiabrando, F., Donadio, E., Rinaudo, F., 2015. SfM for orthophoto generation: a winning approach for cultural heritage knowledge. ISPRS - International Archives of the Photogrammetry, Remote Sensing and Spatial Information Sciences XL-5/W7, 91-98.

Colomina, I., Molina, P., 2014. Unmanned aerial systems for photogrammetry and remote sensing: A review. ISPRS Journal of Photogrammetry and Remote Sensing 92, 79-97.

Fraser, C., 2013. Automatic Camera Calibration in Close Range Photogrammetry. Photogrammetric Engineering \& Remote Sensing 79, 381-388.

Fritsch, D., Becker, S., Rothermel, M., 2013a. Modeling Façade Structures Using Point Clouds From Dense Image Matching. Proceedings of the Intl. Conf. on Advances in Civil, Structural and Mechanical Engineering 57-64.

Fritsch, D., Cramer, M., Haala, N., 2013b. UAV im Einsatz für die Datenerfassung beim LGL BW Abschlussbericht. Stuttgart.

Grussenmeyer, P., Hanke, K., Streilein, A., 2002. Architectural Photogrammety, in: Kasser, M., Egels, Y. (Eds.), Digital Photogrammetry. Taylor \& Francis, pp. 300-339.

Hirschmüller, H., 2011. Semi-Global Matching Motivation, Developments and Applications. Photogrammetric Week 173184.

Luhmann, T., Fraser, C., Maas, H.G., 2016. Sensor modelling and camera calibration for close-range photogrammetry. ISPRS Journal of Photogrammetry and Remote Sensing 115, 37-46.

Murtiyoso, A., Grussenmeyer, P., Freville, T., 2017. Close Range UAV Accurate Recording and Modeling of St-Pierre-leJeune Neo-Romanesque Church in Strasbourg (France). ISPRS - International Archives of Photogrammetry, Remote Sensing and Spatial Information Sciences XLII-2/W3, 519-526.

Murtiyoso, A., Grussenmeyer, P., Koehl, M., Freville, T., 2016. Acquisition and processing experiences of close range UAV images for the 3D modeling of heritage buildings, in: Ioannides,
M. et al. (Eds.), Digital Heritage. Progress in Cultural Heritage: Documentation, Preservation, and Protection: 6th International Conference, EuroMed 2016, Nicosia, Cyprus, October 31 November 5, 2016, Proceedings, Part I. Springer International Publishing, pp. 420-431.

Nex, F., Remondino, F., 2014. UAV: platforms, regulations, data acquisition and processing, in: Remondino, F., Campana, S. (Eds.), 3D Recording and Modelling in Archaeology and Cultural Heritage: Theory and Best Practices. Archaeopress, Oxford, England, pp. 73-86.

Nony, N., De Luca, L., Godet, A., Pierrot-Deseilligny, M., Remondino, F., Van Dongen, A., Vincitore, M., 2012. Protocols and assisted tools for effective image-based modeling of architectural elements. Progress in Cultural Heritage Preservation 7616 LNCS, 432-439.

Pierrot-Deseilligny, M., De Luca, L., Remondino, F., 2011. Automated image-based procedures for accurate artifacts 3D modeling and orthoimage generation. Proceedings of the XXIIIrd International CIPA Symposium.

Pierrot-Deseilligny, M., Paparoditis, N., 2006. A multiresolution and optimization-based image matching approach: An application to surface reconstruction from SPOT5-HRS stereo imagery. ISPRS - International Archives of the Photogrammetry, Remote Sensing and Spatial Information Sciences XXXVI.

Reich, M., Wiggenhagen, M., Muhle, D., 2012. Filling the holes - Potential of UAV-based photogrammetric façade modelling. Tagungsband des 15. 3D-NordOst Workshops der GFaI.

Remondino, F., 2014. Photogrammetry - Basic theory, in: Remondino, F., Campana, S. (Eds.), 3D Recording and Modelling in Archaeology and Cultural Heritage: Theory and Best Practices. Archaeopress, Oxford, England, pp. 63-72.

Remondino, F., Barazzetti, L., Nex, F., Scaioni, M., Sarazzi, D. 2011. UAV photogrammetry for mapping and 3D modeling current status and future perspectives. ISPRS - International Archives of the Photogrammetry, Remote Sensing and Spatial Information Sciences XXXVIII, 25-31.

Remondino, F., Del Pizzo, S., Kersten, T.P., Troisi, S., 2012. Low-cost and open-source solutions for automated image orientation-a critical overview. Progress in Cultural Heritage Preservation 7616 LNCS, 40-54.

Remondino, F., Fraser, C., 2006. Digital camera calibration methods: considerations and comparisons. ISPRS International Archives of Photogrammetry, Remote Sensing and Spatial Information Sciences 36, 266-272.

Remondino, F., Spera, M.G., Nocerino, E., Menna, F., Nex, F., 2014. State of the art in high density image matching. The Photogrammetric Record 29, 144-166.

Remondino, F., Spera, M.G., Nocerino, E., Menna, F., Nex, F., Gonizzi-Barsanti, S., 2013. Dense image matching: Comparisons and analyses. Proceedings of the Digital Heritage 2013 1, 47-54.

Wenzel, K., Rothermel, M., Fritsch, D., Haala, N., 2013. Image acquisition and model selection for multi-view stereo. ISPRS International Archives of the Photogrammetry, Remote Sensing and Spatial Information Sciences XL, 251-258. 